\title{
Synthesis and Characterization of Strontium Titanate Nanoparticles as Potential High Temperature Oxygen Sensor Material
}

\author{
Florian Voigts,, ${ }^{1}$ Tanja Damjanović,, ${ }^{2}$ Günter Borchardt, ${ }^{2}$ Christos Argirusis, ${ }^{2}$ \\ and Wolfgang Maus-Friedrichs ${ }^{1}$ \\ ${ }^{1}$ Institut für Physik und Physikalische Technologien, Technische Universität Clausthal, Leibnizstraße 4, \\ 38678 Clausthal-Zellerfeld, Germany \\ ${ }^{2}$ Institut für Metallurgie, Technische Universität Clausthal, Robert-Koch-Straße 42, \\ 38678 Clausthal-Zellerfeld, Germany
}

Received 13 March 2006; Revised 18 May 2006; Accepted 6 June 2006

\begin{abstract}
We present a simple and highly reproductive method for the preparation of thin films consisting of strontium titanate nanoparticles. The films are produced by spin coating of a sol on silicon targets and subsequent annealing under ambient conditions. Analysis by atomic force microscopy shows particles with typical sizes between $10 \mathrm{~nm}$ and $50 \mathrm{~nm}$. X-ray photoelectron spectroscopy displays a stoichiometry of the films as anticipated from preliminary experiments with strontium titanate single crystals. Metastable-induced electron spectroscopy and ultraviolet photoelectron spectroscopy are used as tools to give evidence to the similar electronic properties of nanoparticle film and single crystal. These results support the prospect for an application of the nanoparticle films as high temperature oxygen sensor with superior properties.
\end{abstract}

Copyright (c) 2006 Florian Voigts et al. This is an open access article distributed under the Creative Commons Attribution License, which permits unrestricted use, distribution, and reproduction in any medium, provided the original work is properly cited.

\section{INTRODUCTION}

Donor doped $\mathrm{SrTiO}_{3}$ (STO) is well known for its capability as resistive high temperature oxygen sensor [1-4]. STO is stable without recrystallization over a wide temperature range between $104 \mathrm{~K}$ and about $2300 \mathrm{~K}$ [5]. Furthermore, STO accepts very high donor dopant concentrations up to $30 \%$ without any phase transition thus allowing to vary the sensor capacity over a wide range $[6,7]$. Especially very porous donor doped STO was found to be of interest, because it provides quick response times in contrast to STO crystals. 0.5 at.- $\% \mathrm{Nb}$-doped STO layers, for example, show response times around $30-40 \mathrm{~ms}$ at $1130 \mathrm{~K}$ [1]. These response times can be improved even further if the particle size is decreased. Meyer and Waser found, that STO crystalline material with grain sizes of about $1 \mu \mathrm{m}$ provides response times of about $10 \mathrm{~ms}$ [2].

Response times in the range of ms are necessary for most applications, for example, for oxygen sensors in car exhaust management. Therefore, the donor doped STO nanocrystalline particles may be of relevance in future high temperature oxygen sensor applications.
Sol-gel is a powerful method for preparation of nanoscaled oxide powders and nanoscale thin films for a variety of applications. It enables processing of refractory oxide layers at temperatures as low as $370 \mathrm{~K}$ in some special cases [8]. Materials with different compositions can be easily obtained in the form of glasses, fibers, ceramic powders, and thin films [9]. Multilayers are also easily made by means of sol-gel methods [10].

In this study we report a sol-gel route for the production of undoped and donor doped STO nanostructured thin films for sensor applications. We characterized these films by microscopic and electron spectroscopic methods.

\section{EXPERIMENTAL}

\subsection{STO-sol and STO coatings preparations}

STO sols were synthesized by means of a sol-gel method starting from alkoxides of the corresponding metals. The reagent grade chemicals used were $\operatorname{Sr}\left(\mathrm{C}_{2} \mathrm{H}_{3} \mathrm{O}_{2}\right)_{2}(99.995 \%$ $\mathrm{Sr}$-Sigma-Aldrich) and $\mathrm{Ti}\left(\mathrm{OC}_{4} \mathrm{H}_{9}\right)_{4} \quad(97 \% \mathrm{Ti}-$ SigmaAldrich). First, Sr-acetate was dissolved in concentrated 
acetic acid under addition of small amounts of acetyl acetone which acts as a complexing agent. The dissolution of $\mathrm{Sr}$ acetate was performed on a magnetic stirrer under continuous heating. After a clear solution of Sr-acetate was obtained Ti-butoxide was added dropwise to the solution.

For the preparation of $\mathrm{Nb}$-doped STO, sol Nb( $\left.\mathrm{C}_{2} \mathrm{H}_{3} \mathrm{O}_{2}\right)_{5}$ (99.9\% Nb-Strem Chemicals) was weighed and added with a syringe to an aliquot of the aforementioned undoped STO sol. In order to achieve the optimal viscosity of the final sol to be spin coated, we continued heating of the Nb-doped STO sol under stirring. The final sol was clear and stable on storage.

STO layers were applied on Si-wafers by means of spin coating upon addition of 20,50 , and $60 \mu \mathrm{l}$ of the STO sol, followed by spinning at $12000 \mathrm{~s}^{-1}$ for 15 seconds. After deposition, the layers were left in ambient air for 1 hour and then thermally treated in air according to the following program: $2 \mathrm{~K} / \mathrm{min}$ up to $570 \mathrm{~K}$ with a dwell of 30 minutes, and further with $3 \mathrm{~K} / \mathrm{min}$ up to $1000 \mathrm{~K}$ with a 1 -hour dwell. The samples were cooled down to room temperature at a cooling rate of $3 \mathrm{~K} / \mathrm{min}$.

\subsection{STO particle characterization}

The spectroscopic measurements were carried out using an ultrahigh vacuum (UHV) apparatus with a base pressure of $5 \times 10^{-9} \mathrm{~Pa}$, which has been described in detail previously [11].

Electron spectroscopy is performed with a hemispherical analyzer (VSW HA100) in combination with a source for metastable helium atoms $\left(\mathrm{He}^{*}\right)$ and ultraviolet photons ( $\mathrm{HeI}$ line) for metastable induced electron spectroscopy (MIES) and ultraviolet photoelectron spectroscopy (UPS) as well as with a commercial non-monochromatic X-ray source (Specs RQ20/38C) for X-ray photoelectron spectroscopy (XPS).

During XPS, X-ray photons hit the surface under an angle of $80^{\circ}$ to the surface normal, illuminating a spot with a diameter of a few millimeters. For the measurements shown here we used the $\mathrm{Mg} \mathrm{K} \alpha$ and $\mathrm{Al} \mathrm{K}$ lines with photon energies of $1253.7 \mathrm{eV}$ and $1486.7 \mathrm{eV}$. Emitted electrons were analyzed by the hemispherical analyzer under $10^{\circ}$ to the surface normal with a resolution of $1.1 \mathrm{eV}$. All XPS spectra are displayed as a function of the electron binding energy with respect to the Fermi level. Therefore, an apparent shift of all Auger lines by $233.0 \mathrm{eV}$ occurs when switching between excitation sources.

For quantitative XPS analysis, the photoelectron peak area is calculated by mathematical fitting with Gauss-type profiles using OriginPro $7 \mathrm{G}$ including the PFM fitting module. Photoelectric cross sections as calculated by Scofield [12] and inelastic mean free paths from the NIST database [13] are taken into account when calculating stoichiometry. Furthermore, the transmission function of our hemispherical analyzer is included into the calculation.

MIES and UPS are performed applying a cold cathode gas discharge via a two-stage pumping system. Here, metastable $\mathrm{He}^{*}$ atoms and HeI photons are produced. The ratio between $\mathrm{He}^{*} 2^{3} \mathrm{~S}$ and $\mathrm{He}^{*} 2^{1} \mathrm{~S}$ amounts to $7: 1$ [14] at least. Therefore, no contribution of the $\mathrm{He}^{*} 2^{1} \mathrm{~S}$-surface interaction can be detected. A time-of-flight technique is used to separate electrons emitted by $\mathrm{He}^{*}$ (MIES) and HeI (UPS) interaction with the surface. The mixed $\mathrm{He}^{*} / \mathrm{HeI}$ beam strikes the surface under an angle of $45^{\circ}$ and illuminates an area of about $2 \mathrm{~mm}$ diameter on the sample surface. MIES and UPS spectra are simultaneously recorded by the hemispherical analyzer with a resolution of $220 \mathrm{meV}$ under normal emission within 130 second.

Metastable $\mathrm{He}^{*}$ atoms may interact with the sample via different mechanisms depending on surface electronic structure and work function $[15,16]$. In this work, we will only discuss the relevant processes.

During Auger deexcitation (AD), an electron from the sample surface fills the 1 s orbital of the impinging $\mathrm{He}^{*}$. Simultaneously, the He 2s electron is emitted carrying the excess energy. The resulting spectra directly display the surface density of states (SDOS). The excitation potential of the $\mathrm{He}^{*}$ amounts to $19.8 \mathrm{eV}$ [15-18]. Because the $\mathrm{He}^{*}$ atoms interact with the surface in distances typically between 0.3 and $0.5 \mathrm{~nm}$ in front of it, MIES is extremely surface sensitive and displays the SDOS of the uppermost layer of the sample only. To distinguish surface from bulk effects, AD-MIES and UPS can be compared directly.

For STO, a different interaction takes place: the $2 \mathrm{~s}$ electron of the impinging $\mathrm{He}^{*}$ is resonantly transferred into the surface of the sample and localizes at near surface Ti $3 \mathrm{~d}$ states. Subsequently, a Ti $3 \mathrm{~d}$ electron fills the hole in $\mathrm{He}^{+}$1s in an interatomic Auger neutralization (AN) process, followed by the emission of an $\mathrm{O}(2 \mathrm{p})$ surface electron carrying the excess energy. The energy of the resulting MIES peak is shifted by $1.2 \mathrm{eV}$ toward higher binding energies compared to AD due to a diminished local ionization potential. A detailed discussion of this process is given in [19].

Oxygen incorporation into STO most strongly depends on the dissociation probability in the vicinity of the surfaces. This probability depends on the very outermost surface wave functions which are most sensitively probed by MIES.

All MIES and UPS spectra are displayed as a function of the electron binding energy with respect to the Fermi level. The surface work function can be determined from the left onset of the MIES or the UPS spectra with an accuracy of $0.1 \mathrm{eV}$.

The silicon samples bearing the STO nanoparticles are mounted in a molybdenum sample holder and transferred into the UHV via a transfer system. Afterwards, the sample is cleaned from surface contamination by heating to about $850 \mathrm{~K}$ before measuring.

Atomic force microscopy (AFM) measurements are performed with a digital instruments dimension 3100 AFM in tapping mode using a silicon cantilever. The AFM is operated under ambient conditions. AFM images are analyzed concerning particle size distribution and surface roughness with SPIP 3.2 from image metrology.

Auger depth profiles were used to measure the thickness of the STO layers on the silicon by sputtering with argon under control of a PHI Auger microprobe system. These 


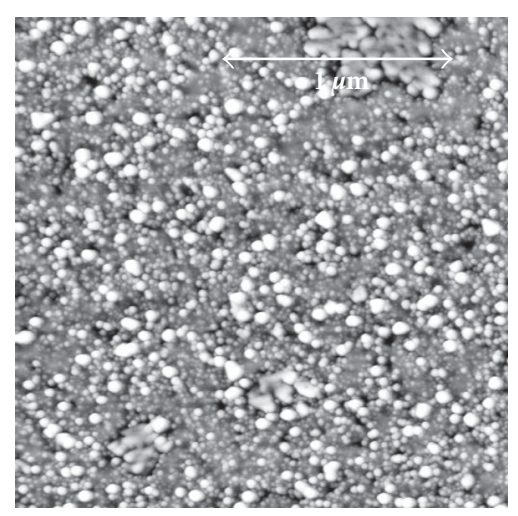

(a)

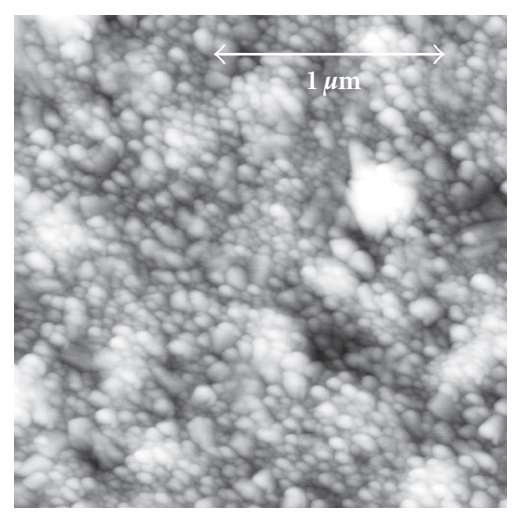

(b)

Figure 1: AFM images $\left(2 \times 2 \mu \mathrm{m}^{2}\right)$ of an (a) undoped and (b) Nbdoped $\mathrm{SrTiO}_{3}$ nanoparticle film on a $\mathrm{Si}(100)$ substrate.

measurements were carried out in a separate vacuum chamber.

\section{RESULTS}

Figure 1 shows the AFM images of a STO undoped (Figure $1(\mathrm{a})$ ) and $\mathrm{Nb}$-doped (Figure 1(b)) nanoparticle film, which was produced via the sol-gel route described in Section 2. After deposition the samples were transferred to the microscope immediately. We evaluate an average size of $25 \mathrm{~nm}$ with a full width at half maximum (FHWM) of $20 \mathrm{~nm}$ for the undoped particles and an average size of $50 \mathrm{~nm}$ with a FHWM of $30 \mathrm{~nm}$ for the donor doped particles. The films are thinner than $100 \mathrm{~nm}$ in both cases, which was evaluated by Auger depth profile analysis. The particle sizes and their distributions can be reproduced with high accuracy applying the synthesis parameters described in Section 2. The surfaces possess roughnesses of about $3.8 \mathrm{~nm}$ for the undoped and $6.3 \mathrm{~nm}$ for the doped films (root mean square corresponding to ISO $4287 / 1$ ). Heating to temperatures substantially higher than described in Section 2.1 does not change neither the size nor the distribution of the nanoparticles.

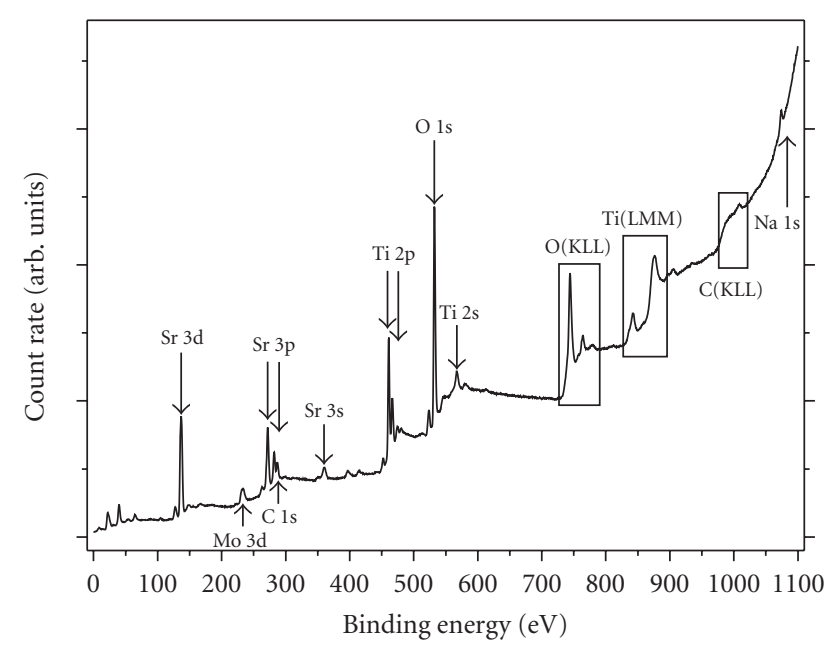

(a)

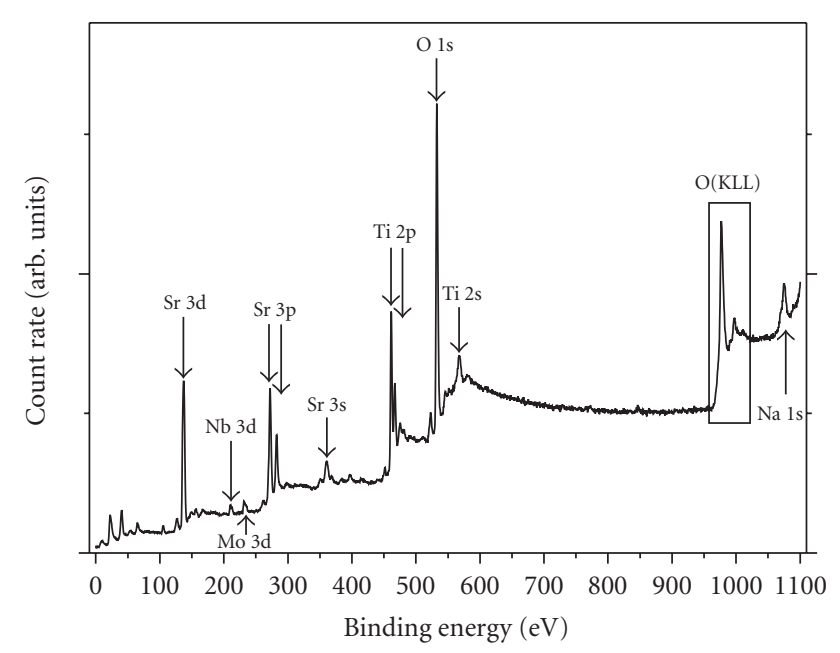

(b)

FIgure 2: (a) XPS spectrum using $\mathrm{Mg} \mathrm{K} \mathrm{K}_{\alpha}$ excitation of an undoped $\mathrm{SrTiO}_{3}$ nanoparticle film on a $\mathrm{Si}(100)$ substrate, (b) XPS spectrum using $\mathrm{Al} \mathrm{K} \alpha$ excitation of a $\mathrm{Nb}$-doped $\mathrm{SrTiO}_{3}$ nanoparticle film on a $\mathrm{Si}(100)$ substrate. $\mathrm{O}(\mathrm{KLL}), \mathrm{Ti}(\mathrm{LMM})$, and $\mathrm{C}(\mathrm{KLL})$ are shifted by $233.0 \mathrm{eV}$ towards higher binding energies compared to Figure 2(a).

Figure 2 shows the XPS spectra of the undoped (Figure 2(a)) and Nb-doped (Figure 2(b)) STO nanoparticle films shown in Figure 1. The samples were immediately inserted into the UHV after the microscopy. The peak areas are analyzed as described in Section 2 and are compared with the corresponding ones for stoichiometric STO. For the undoped particles we find a composition near to STO but with a Sr concentration about 5\% lower than the $\mathrm{Ti}$ concentration. Comparable values are found for the doped particles with a $\mathrm{Nb}$ concentration of about 1 at.- $\%$. This corresponds well to the value expected from preparation.

Besides the peaks corresponding to STO we observe Mo, $\mathrm{C}$, and Na signals. Mo is due to the sample holder and is not 


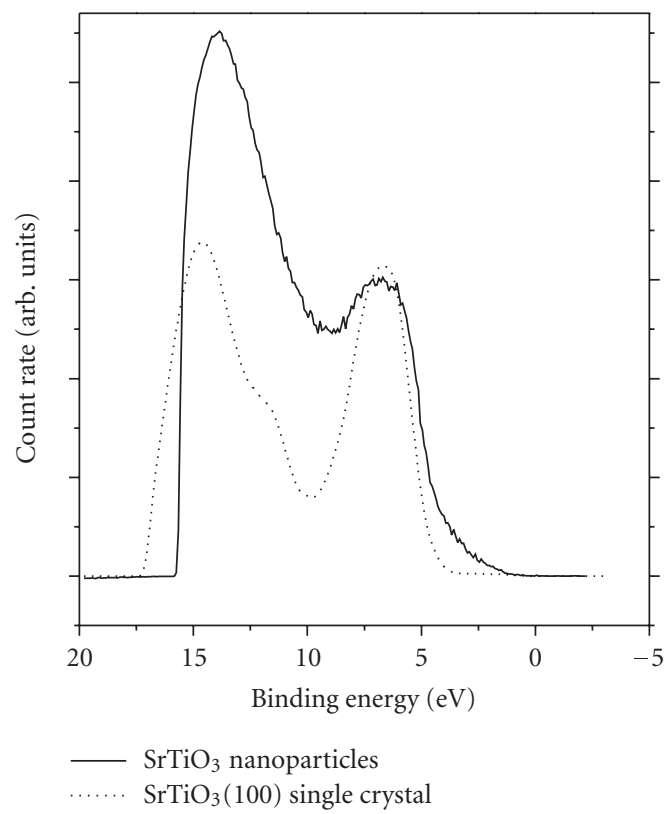

(a)

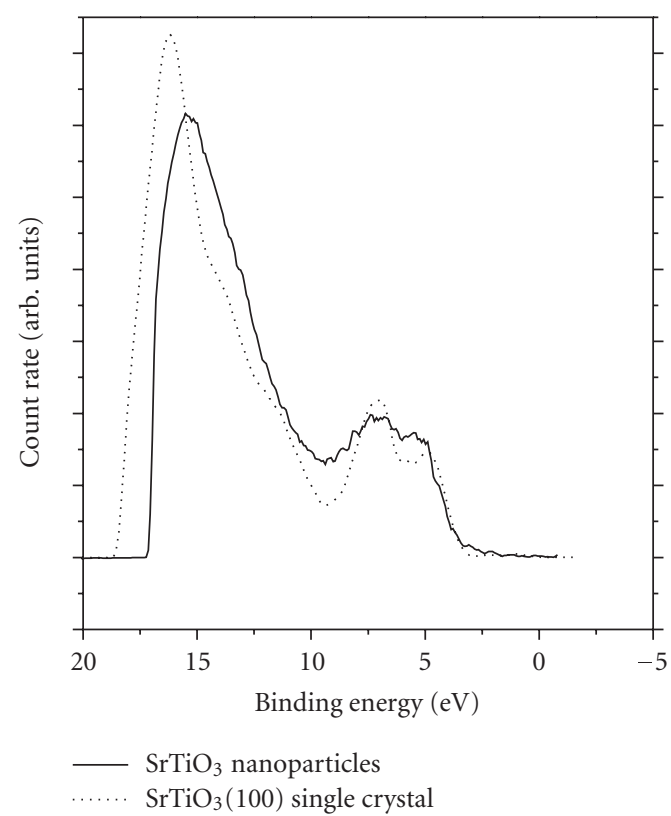

(b)

Figure 3: (a) MIES and (b) UPS spectra of an $\mathrm{SrTiO}_{3}$ nanoparticle film on an $\mathrm{Si}(100)$ substrate (solid line) compared to the corresponding spectra of $\mathrm{SrTiO}_{3}(100)$ single crystals (dotted line).

found on the sample surface. $\mathrm{C}$ is typically due to a surface contamination with carbon containing species like $\mathrm{CO}_{2}$ or $\mathrm{CO}_{3}^{2-}$. The $\mathrm{Na}$ contamination is mostly due to $\mathrm{NaCl}$ residues from the deposition process.

It is well known from literature that donor doped STO crystals heated under ambient conditions loose Sr [20, 21]. We assume that the annealing during the synthetisation process induces similar processes on the nanoparticle surfaces, thus reducing the $\mathrm{Sr}$ concentration.

Figure 3 shows MIES (Figure 3(a)) and UPS spectra (Figure 3(b)) of the undoped nanoparticle film discussed above compared with corresponding spectra for $\mathrm{SrTiO}_{3}(100)$ single crystals $[19,22,23]$. Nb-doped nanoparticles show similar spectra which are therefore not shown here. The spectra are displayed as a function of the binding energy $E_{B}$ with the Fermi level at $E_{B}=0 \mathrm{eV}$. The contributions beyond $E_{B} \approx 10 \mathrm{eV}$ are induced by secondary electrons and will not be discussed here. The MIES spectra show one peak at $E_{B}=6.5 \mathrm{eV}$ being induced by the localized AN process described in Section 2 [19]. This peak is well known and corresponds to the ionization of non-hybridized $\mathrm{O}(2 \mathrm{p})$ of the $\mathrm{SrTiO}_{3}(100)$ surface, which is terminated by a $\mathrm{TiO}_{2}$ layer [24]. The valence band maximum (VBM) of $\mathrm{SrTiO}_{3}(100)$ single crystals is found around $E_{B}=3.2 \mathrm{eV}$ while the VBM on the STO particle film reaches to $E_{B} \approx 1.9 \mathrm{eV}$.

UPS shows a double peak structure at $E_{B}=7.0 \mathrm{eV}$ and $E_{B}=4.9 \mathrm{eV}$. The peak at higher binding energy corresponds to the ionization of $\mathrm{O}(2 \mathrm{p})$ orbitals being hybridized with $\mathrm{Ti}$ $3 \mathrm{~d}$, while the peak at lower binding energy is due to nonhybridized $\mathrm{O}(2 \mathrm{p})$. The $\mathrm{VBM}$ is found around $E_{B} \approx 3.2 \mathrm{eV}$ for the single crystal and the film.

\section{DISCUSSION}

Applying the simple synthesis procedure described in Section 2 , films containing nanoscale particles are produced easily. These particles consist of $\mathrm{Sr}, \mathrm{Ti}$, and $\mathrm{O}$ with near STO stoichiometry. The reduced $\mathrm{Sr}$ concentration is most probably due to the applied heating procedure described in Section 2. It is well known that an Sr loss occurs during heating STO single crystals in oxygen containing atmospheres. UPS and MIES show a quite good similarity between the particle film and well-defined $\mathrm{SrTiO}_{3}(100)$ surfaces. The latter one is known to show good high temperature oxygen sensor capabilities. The (110) and (111) surfaces are known to show the same electronic structures.

The similarity between the UPS spectra proves that the surface density of states of the nanoparticles is very similar to the one for STO single crystals. It is known that the electronic and geometric structure of STO does not change even for large amount of Sr vacancies inside the crystal $[6,7]$.

The interaction of the impinging $\mathrm{He}^{*}$ atoms approaching the surface with thermal velocity probes only the very outermost surface wave functions. These wave functions are also responsible for the interaction of sensor surfaces with impinging oxygen or ambient atmospheres molecules. In this sense MIES is a very reasonable and powerful tool to investigate the heterogenous reactivity of the sensor surface. The observed similarity between the MIES spectra for single crystal and the film is of great relevance for future sensor applications. It suggests that the nanocrystalline film can be expected to behave similarly to STO single crystals. Furthermore, in MIES contributions within the STO band gap are 
observed. These are likely due to surface defects, which will enhance the surface reactivity further.

It was shown previously that typical oxygen diffusion lengths in STO amount to several $10 \mathrm{~nm}$ in a diffusion time of $10 \mathrm{~ms}$ [25]. The oxygen diffusion on the nanoparticles surfaces may be expected to be much faster than inside the crystals. For $\mathrm{ZrO}_{2}$ it was found that the surface diffusion is about $10^{4}$ times faster than the diffusion into the bulk [26] This means that nanoparticles with a diameter of several $10 \mathrm{~nm}$ can be expected to provide a very high signal, because all particles contribute to the oxygen sensor signal with their complete volume.

\section{SUMMARY}

Undoped and 1 at.-\% Nb-doped nanoparticle containing films with near STO stoichiometry were produced applying a simple sol-gel procedure. The undoped particles show a mean diameter of $25 \mathrm{~nm}$ with a FHWM of $20 \mathrm{~nm}$ while the doped particles show a mean diameter of $50 \mathrm{~nm}$ with a FHWM of $30 \mathrm{~nm}$. The films are electronically very similar to STO single crystals. Their surface provides a reactivity which is expected to be even higher than the ones for single crystal surfaces. On the one hand, the particle size of several $10 \mathrm{~nm}$ is sufficiently high to provide stable electronic conditions of the particles. On the other hand, the size is low enough to allow complete oxygen diffusion into the particles within $10 \mathrm{~ms}$. It can be expected that oxygen diffusion on the particles surface is very fast. Therefore, the produced nanocrystalline STO is a highly promising candidate for a very fast and very sensitive high temperature oxygen sensor.

\section{ACKNOWLEDGMENTS}

The authors gratefully acknowledge support from the Deutsche Forschungsgemeinschaft (DFG) under contract numbers. Ma 1893/9-1 and Ar 248/3-1. We wish to express our gratitude to Dipl.-Ing. Peter Cyris for the accomplishment of the AFM and Auger depth profile measurements.

\section{REFERENCES}

[1] W. Menesklou, H.-J. Schreiner, K. H. Härdtl, and E. IversTiffée, "High temperature oxygen sensors based on doped $\mathrm{SrTiO}_{3}$," Sensors and Actuators B: Chemical, vol. 59, no. 2, pp. 184-189, 1999.

[2] R. Meyer and R. Waser, "Resistive donor-doped $\mathrm{SrTiO}_{3}$ sensors: I, basic model for a fast sensor response," Sensors and Actuators B: Chemical, vol. 101, no. 3, pp. 335-345, 2004.

[3] A. Gunhold, L. Beuermann, K. Gömann, et al., "Study of the electronic and atomic structure of thermally treated $\mathrm{SrTiO}_{3}(110)$ surfaces," Surface and Interface Analysis, vol. 35, no. 12, pp. 998-1003, 2003.

[4] A. Gunhold, K. Gömann, L. Beuermann, V. Kempter, G. Borchardt, and W. Maus-Friedrichs, "Changes in the surface topography and electronic structure of $\mathrm{SrTiO}_{3}\left(\begin{array}{lll}1 & 1 & 0\end{array}\right)$ single crystals heated under oxidizing and reducing conditions," Surface Science, vol. 566-568, no. 1-3, part 1, pp. 105-110, 2004.
[5] J. Padilla and D. Vanderbilt, "Ab initio study of $\mathrm{SrTiO}_{3}$ surfaces," Surface Science, vol. 418, no. 1, pp. 64-70, 1998.

[6] R. Moos, T. Bischoff, W. Menesklou, and K. H. Härdtl, "Solubility of lanthanum in strontium titanate in oxygen-rich atmospheres," Journal of Materials Science, vol. 32, no. 16, pp. 4247-4252, 1997.

[7] S. Steinsvik, R. Bugge, J. Gjønnes, J. Taftø, and T. Norby, "The defect structure of $\mathrm{SrTi}_{1-\mathrm{x}} \mathrm{Fe}_{\mathrm{x}} \mathrm{O}_{3-\mathrm{y}}(\mathrm{x}=0-0.8)$ investigated by electrical conductivity measurements and electron energy loss spectroscopy (EELS)," Journal of Physics and Chemistry of Solids, vol. 58, no. 6, pp. 969-976, 1997.

[8] S. Sakka, "Current sol-gel activities in Japan," Journal of SolGel Science and Technology, vol. 37, no. 2, pp. 135-140, 2006.

[9] M. L. Zheludkevich, I. Miranda Salvado, and M. G. S. Ferreira, "Sol-gel coatings for corrosion protection of metals," Journal of Materials Chemistry, vol. 15, no. 48, pp. 5099-5111, 2005.

[10] W. Hu, C. Yang, W. Zhang, and Y. Qiu, "Dielectric characteristics of sol-gel-derived BST/BSLaT/BST multilayer," Journal of Sol-Gel Science and Technology, vol. 36, no. 3, pp. 249-255, 2005.

[11] M. Frerichs, F. Voigts, and W. Maus-Friedrichs, "Fundamental processes of aluminium corrosion studied under ultra high vacuum conditions," to appear in Applied Surface Science.

[12] J. H. Scofield, "Hartree-Slater subshell photoionization crosssections at 1254 and $1487 \mathrm{eV}$," Journal of Electron Spectroscopy and Related Phenomena, vol. 8, no. 2, pp. 129-137, 1976.

[13] "NIST Electron Inelastic-Mean-Free-Path Database 1.1," http://www.nist.gov/srd/nist71.htm.

[14] J. Günster, Elektronenspektroskopische Untersuchungen an Isolierenden Schichten auf W (110), Al (111) und Si (100), Ph.D. thesis, Technische Universität Clausthal, Clausthal, Germany, 1996.

[15] Y. Harada, S. Masuda, and H. Ozaki, "Electron spectroscopy using metastable atoms as probes for solid surfaces," Chemical Reviews, vol. 97, no. 6, pp. 1897-1952, 1997.

[16] H. Morgner, "The characterization of liquid and solid surfaces with metastable helium atoms," Advances in Atomic, Molecular, and Optical Physics, vol. 42, pp. 387-488, 2000.

[17] G. Ertl and J. Küppers, Low Energy Electrons and Surface Chemistry, VCH, Weinheim, Germany, 1985.

[18] V. Kempter, "Study of insulator surfaces with the Metastable Impact Electron Spectroscopy (MIES)," Materials Science Forum, vol. 239-241, pp. 621-628, 1997.

[19] W. Maus-Friedrichs, M. Frerichs, A. Gunhold, S. Krischok, V. Kempter, and G. Bihlmayer, "The characterization of $\mathrm{SrTiO}_{3}(0$ 0 1) with MIES, UPS(HeI) and first-principles calculations," Surface Science, vol. 515, no. 2-3, pp. 499-506, 2002.

[20] K. Szot, W. Speier, J. Herion, and Ch. Freiburg, "Restructuring of the surface region in $\mathrm{SrTiO}_{3}$," Applied Physics A: Materials Science \& Processing, vol. 64, no. 1, pp. 55-59, 1997.

[21] K. Szot and W. Speier, "Surfaces of reduced and oxidized $\mathrm{SrTiO}_{3}$ from atomic force microscopy," Physical Review B: Condensed Matter and Materials Physics, vol. 60, no. 8, pp. 5909-5926, 1999.

[22] A. Gunhold, L. Beuermann, M. Frerichs, et al., "Island formation on 0.1 at.\% La-doped $\mathrm{SrTiO}_{3}\left(\begin{array}{lll}1 & 0 & 0\end{array}\right)$ at elevated temperatures under reducing conditions," Surface Science, vol. 523, no. 1-2, pp. 80-88, 2003.

[23] V. E. Henrich and P. A. Cox, The Surface Science of Metal Oxides, Cambridge University Press, Cambridge, UK, 1994. 
[24] P. A. W. van der Heide, Q. D. Jiang, Y. S. Kim, and J. W. Rabalais, "X-ray photoelectron spectroscopic and ion scattering study of the $\mathrm{SrTiO}_{3}\left(\begin{array}{lll}0 & 0 & 1\end{array}\right)$ surface," Surface Science, vol. 473, no. 1-2, pp. 59-70, 2001.

[25] K. Gömann, G. Borchardt, M. Schulz, et al., "Sr diffusion in undoped and $\mathrm{La}$-doped $\mathrm{SrTiO}_{3}$ single crystals under oxidizing conditions," Physical Chemistry Chemical Physics, vol. 7, no. 9, pp. 2053-2060, 2005.

[26] H. L. Tuller, "Ionic conduction in nanocrystalline materials," Solid State Ionics, vol. 131, no. 1, pp. 143-157, 2000. 

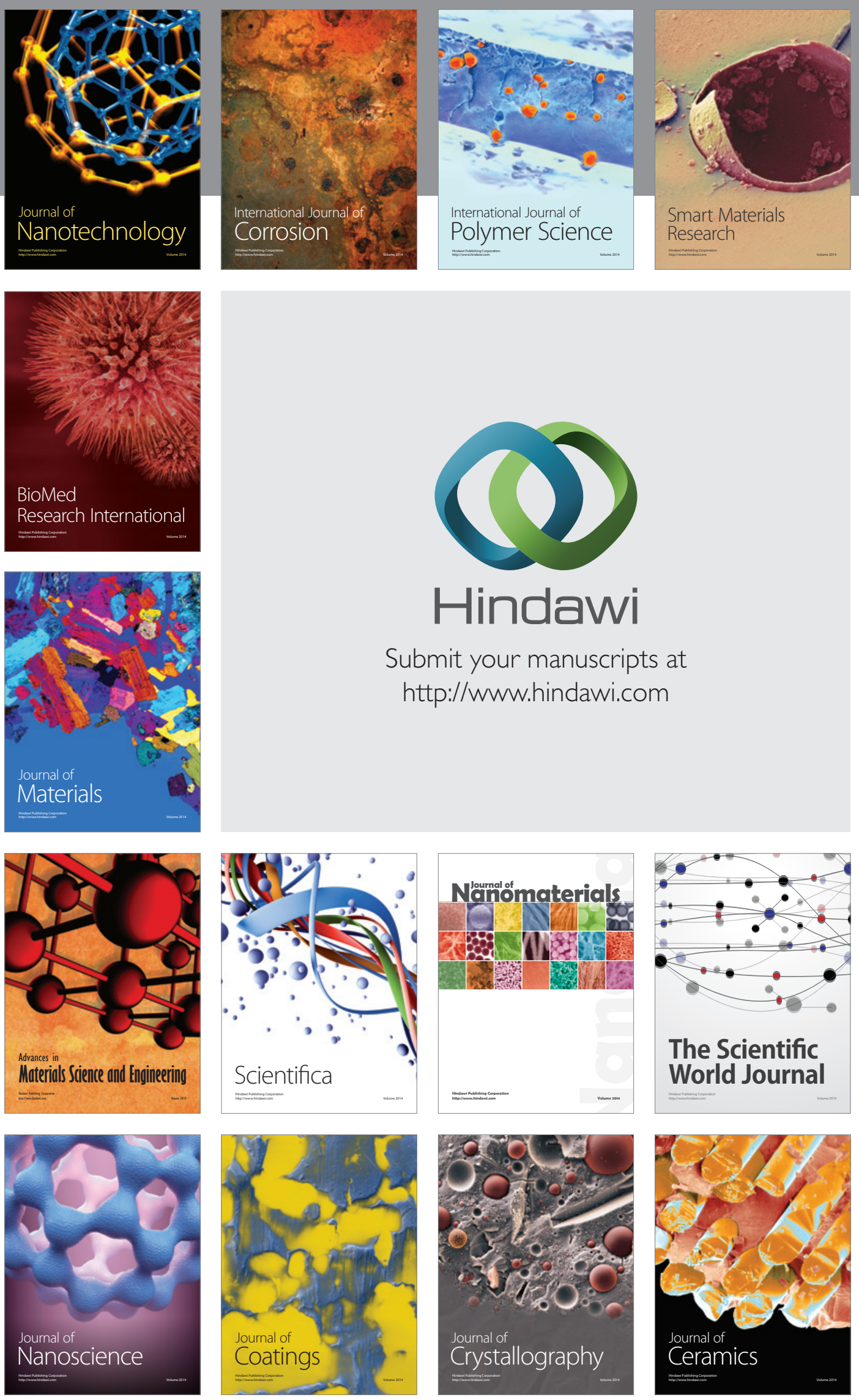

The Scientific World Journal

Submit your manuscripts at

http://www.hindawi.com

\section{World Journal}

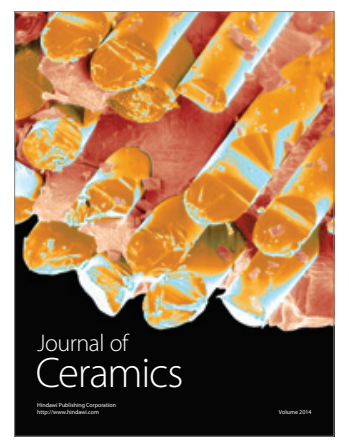

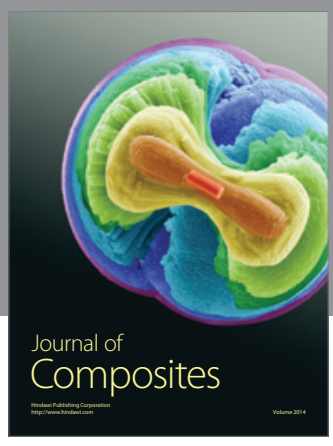
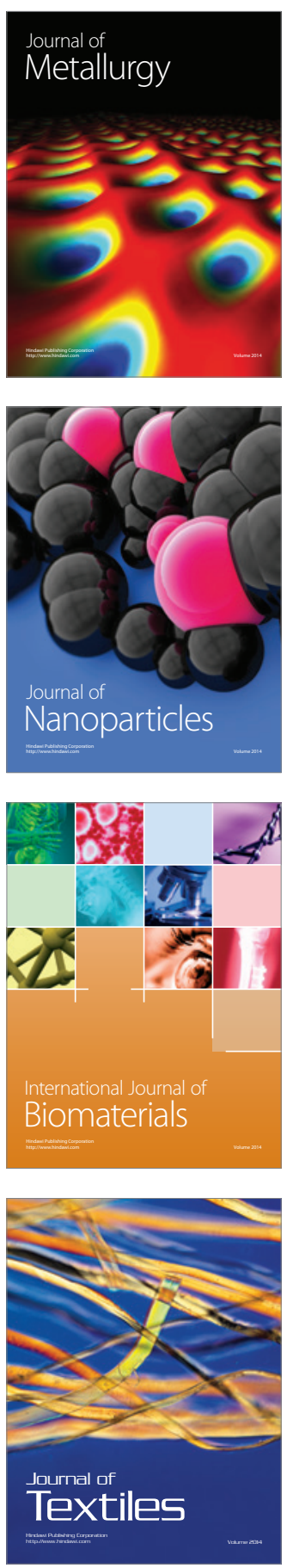